Original article

\title{
Synthesis, characterisation and evaluation of N-mannich bases of 2-substituted Benzimidazole derivatives
}

\author{
Sekar Vinoth Kumar ${ }^{a, *}$, Mohan Raj Subramanian ${ }^{\mathrm{b}}$, Santhosh Kumar Chinnaiyan ${ }^{\mathrm{c}}$ \\ ${ }^{a}$ Department of Pharmaceutical Chemistry, Gokulakrishna College of Pharmacy, Andhra Pradesh 524121, India \\ ${ }^{\mathrm{b}}$ Department of Pharmacology, SSM College of Pharmacy, Tamil Nadu 638312, India \\ ${ }^{\mathrm{c}}$ Department of Pharmaceutical Biotechnology, Srikrupa Institute of Pharmaceutical Sciences, Andhra Pradesh 502277, India
}

\section{A R T I C L E I N F O}

\section{Article history:}

Received 23 August 2013

Accepted 16 November 2013

Available online 11 December 2013

\section{Keywords:}

Benzimidazoles

O-Phenylene diamine

Mannich base

Microbroth dilution

Brine-shrimp lethality

\begin{abstract}
A B S T R A C T
Rationale: Benzimidazoles and its derivatives represent one of the mainly biological active classes of literature.

Aim: In this present study aimed to synthesize $\mathrm{N}$-mannich bases derivatives compounds bearing of 2-substituted benzimidazole moiety, in order to investigate their possible biological activity.

Method: Benzimidazole compounds were prepared from the condensation reaction between ortho phenylene diamine and various acids. Mannich base of newly synthesized Benzimidazole derivatives were synthesized from 2-substituted Benzimidazoles by reacting with secondary amines. The purity of the compounds was ascertained by melting point (m.p) and thin layer chromatography (TLC). Structures of the synthesized compounds were elucidated by spectral data. Antimicrobial assay was performed by microbroth dilution method. Bacterial genomic DNA cleavage was assessed by Agarose gel electrophoresis. Toxicity of the most effective compounds was studied by Brine-shrimp lethality assay.

Result: Among the synthesized compounds, compound 5E (a) and (b) was establish to be the most potent against all tested microorganisms. This two compounds exhibited complete bacterial DNA cleavage and non-toxic.

Conclusion: These results suggest that it an interesting compound compared to the current therapeutic agents and are considered to investigate further for the same.

Copyright @ 2013 , InPharm Association, Published by Reed Elsevier India Pvt. Ltd. All rights reserved.
\end{abstract}

\section{Introduction}

Benzimidazole derivatives are eminent biologically active $\mathrm{N}$ containing heterocycles, ${ }^{1}$ it is well known that benzimidazole derivatives attain antimicrobial analgesic and anti-inflammatory activities, as well as proved to have activities against HIV and cancer. Heterocyclic nucleus and substituted amino group at 1-position of the benzimidazole were reported to be associated with potent antiinflammatory activity. ${ }^{2}$ Therefore it was thought that preparing Mannich base derivatives from 2-substituted benzimidazoles would probably result in compounds of having high biological activities toward many diseases.

The extensively used as drugs such as proton pump inhibitor ${ }^{3}$ (Omeprazole), Antihelmenthetics ${ }^{4}$ (Albendazole), antidopaminergic $^{5}$ (Domperidone), specifically, the 2-substituted analogs of

\footnotetext{
* Corresponding author. Tel.: +91 8099149014.

E-mail addresses: vinogkcp@gmail.com, skredmind7@gmail.com (S. Vinoth Kumar).
}

benzimidazoles are known to be potent biologically active compounds against inflammation, viral and microbial infection. Furthermore, benzimidazole derivatives are structural isosteres of naturally occurring nucleotides, which allows them to interact easily with the biopolymers of the living systems. ${ }^{6}$

Mannich reaction is a condensation between a compound containing atleast one active hydrogen atom, formaldehyde and ammonia, secondary amine have been used as a synthetic tool in the preparation of various therapeutic agents like, fluoxetine as antidepressant agent, ethacrynic acid a high ceiling loop diuretic, benzoquinamide, a high psychotic agent, Ranitidine, Triprolidine an $\mathrm{H}$-receptor antagonist, and Trihexylphenidyl hydrochloride, an antispasmodic. ${ }^{7}$ Mannich bases are physiologically reactive because of the basic function rendering the molecule soluble in aqueous solvent when it is transformed into ammonium salt. ${ }^{8}$ Over the past few decades, Mannich bases of heterocyclic molecules have been grabbing the attention of the synthetic chemists for their wide gamut of biological activities ranging from antibacterial, ${ }^{9}$ anticancer, antiparkinson to anticonvulsant, analgesic, antispasmodic, anti-HIV, anti-malarial as well as intermediates in drug synthesis. ${ }^{10}$ 2-Substituted benzimidazole derivatives, one of the 
most important derivatives of benzimidazole are known to possess varied biological activities. In SAR, the biological activities of benzimidazole compounds depend upon the substitution at the $\mathrm{N}$ 1 or C-2 position. ${ }^{11,12}$ These observations have been guiding for the development of new mannich bases of 2-substituted benzimidazole derivatives and evaluated for antibacterial and anthelmintic activity.

In this present study, we aim to synthesis of a number of Mannich bases derived from substituted benzimidazole, then these compounds were characterized by FT-IR, ${ }^{1} \mathrm{H}$ NMR spectra and their most probable antimicrobial and lethality test.

\section{Materials and methods}

The melting points of the synthesized compounds were determined using a melting point apparatus and are uncorrected. Completion of the reaction and the purity of the synthesized compounds were ascertained by TLC using the solvent system Chloroform and Methanol (9:1) and the spots were detect using UV-Chamber. The synthesized compounds were characterized using MB 3000 series FT-IR Spectrophotometer by KBr-pellet method. ${ }^{1} \mathrm{H}$ NMR spectra was recorded on AMX-400 NMR spectrophotometer at $400 \mathrm{MHz}$ using DMSO-d6 as the solvent and tetra methyl silane (TMS) as an internal standard. The chemical shifts are articulated in $\delta \mathrm{ppm}$. Synthesis of the intermediate and target compounds was accomplished according to the steps deplicted in Scheme 1.

\subsection{Synthesis of mannich bases}

\subsubsection{General procedure for the synthesis of 2-substituted} benzimidazole derivatives

A solution of substituted acid $(0.01 \mathrm{~mol})$ and 0 -phenylene diamine $(0.01 \mathrm{~mol})$ in $20 \mathrm{ml}$ acetic acid was refluxed for $4 \mathrm{~h}$, the precipitate obtained after cooling was recrystallized from ethanol. ${ }^{13}$

\subsubsection{General procedure for the synthesis of mannich bases}

Mannich Bases were prepared by a solution of 2-substituted benzimidazole $(0.005 \mathrm{~mol})$ in $10 \mathrm{ml}$ ethanol, $0.005 \mathrm{~mol}$ of secondary amine and $0.005 \mathrm{~mol}$ of formaldehyde and then the reaction mixture was refluxed for $8 \mathrm{~h}$. On cooling, the product formed was filtered, dried and recrystallised from Di methyl foramide (DMF). Specific details given to each compounds. ${ }^{13}$

\subsection{Biological activity}

\subsubsection{Antibacterial and antifungal assays}

Minimal inhibitory concentration (MIC, $\mu \mathrm{g} / \mathrm{ml}$ ) is defined as the lowest concentration of target compounds that completely inhibit the growth of bacteria, by means of standard two-fold serial dilution method in 96-well micro-titer plates according to the National Committee for Clinical Laboratory Standards (NCCLS). Ampicillin, and Fluconazole, was used as control drugs for bacteria and fungi respectively. DMSO with inoculation bacterial not medicine was used as positive control to ensure that the solvent had no effect on bacteria growth. All the bacteria and fungi growth was monitored visually and UV-spectrophotometrically and the experiments were performed in triplicate. ${ }^{14}$

2.2.1.1. Preparation of inocula. The microorganisms were subcultured on Mueller Hinton broth (MHA) or Sabouraud dextrose broth (SDA) for bacteria and fungi, respectively, follow by incubation for $24 \mathrm{~h}$ at $37^{\circ} \mathrm{C}$. Inocula were prepared by transferring several colonies of microorganisms to sterile distilled water $(5 \mathrm{ml})$. The suspensions were diluted in sterile distilled water were made to obtain the required working suspensions $\left(1-5 \times 10^{4} \mathrm{CFU} / \mathrm{ml}\right)$.

2.2.1.2. Preparation of plates. The test was performed in 96-well sterile microplates. All the wells received $100 \mu \mathrm{l}$ of MHA (for bacteria) or Sabouraud broth (for fungus) supplemented with $10 \%$ glucose and $0.2 \%$ resazurin. The $100 \mu \mathrm{l}$ of the working solution $(1024,512,256,128,64,32,16,8,4,2,1 \mu \mathrm{g} / \mathrm{ml})$ of $5 \mathrm{~A}$ (a)-5G (b) were added into the wells in rows $A$ to $H$ in column 1 . By using a multichannel pipette, $100 \mu \mathrm{l}$ medium was transferred from column 1 to column 2, and the contents of the wells be mixed glowing. Identical serial 1:2 dilutions were continued through column 10 and $100 \mu \mathrm{l}$ of excess medium was discarded from the wells in column 10. The $100 \mu \mathrm{l}$ of the inoculums suspension was added to the wells in rows A to $\mathrm{H}$ in columns 1to11. Two wells column served as drug free controls. Another two-fold serial dilution of Ampicillin or Fluconazole was used as a positive control against bacteria and fungus, respectively. Each microplate was covered and incubated for $24 \mathrm{~h}$ at $37^{\circ} \mathrm{C}$. Any color changes from purple to pink or colorless were recorded as positive. The lowest concentration at which color change occurred was taken as the MIC value. The average of three values was calculated and that was the MIC for the test material and bacterial or fungal strain. To confirm MIC $s$ and to establish Minimum Bactericidal Concentration (MBC), $20 \mu$ of each culture medium with no visible growth was removed from each well and inoculated in MHA or SDA agar plates. After 16-20 h of aerobic incubation at $37{ }^{\circ} \mathrm{C}$, the number of surviving organisms was determined. MBC was defined as the lowest extract concentration at which $99.9 \%$ of the bacteria were killed. Each experiment was repeated twice.

\subsubsection{DNA Cleavage study}

2.2.2.1. Isolation of DNA. The $24 \mathrm{~h}$ old Escherichia coli culture $(1.5 \mathrm{ml})$ is centrifuged to obtain the pellet, which is then dissolved in $0.5 \mathrm{~mL}$ of lysis buffer (100 mM tris pH 8.0, $50 \mathrm{mM}$ EDTA, 10\% SDS). To this $0.5 \mathrm{ml}$ of saturated phenol was added and incubated at $55^{\circ} \mathrm{C}$

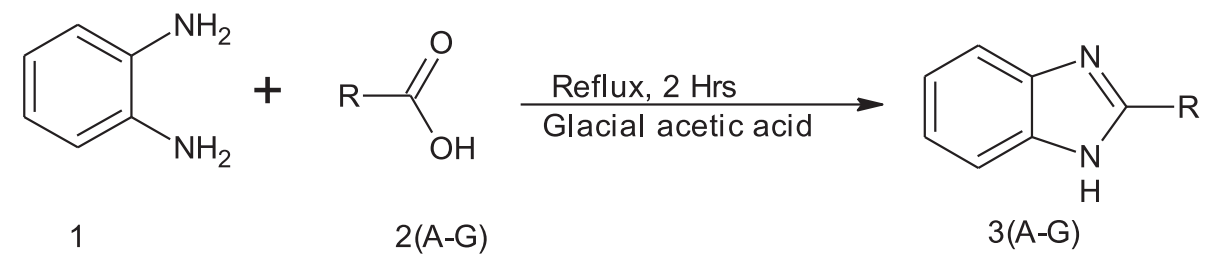

$3 \mathrm{~A}=\mathrm{R}=-\mathrm{H}, 3 \mathrm{~B}=\mathrm{R}=-\mathrm{CH}_{3}, 3 \mathrm{C}=\mathrm{R}=-\mathrm{C}_{6} \mathrm{H}_{5}, 3 \mathrm{D}=\mathrm{R}=-\mathrm{C}_{6} \mathrm{H}_{4}(\mathrm{OH}), 3 \mathrm{E}=\mathrm{R}=-\mathrm{C}_{6} \mathrm{H}_{3}(\mathrm{OH}) \mathrm{SO}_{2} \mathrm{OH}$

$3 \mathrm{~F}=\mathrm{R}=-\mathrm{COOH}, 3 \mathrm{G}=\mathrm{R}=-\mathrm{C}_{6} \mathrm{H}_{4} \mathrm{COOH}$

Scheme 1. Preparation of Manich Base of 2- Substituted Benzimidazole derivatives. 
for $10 \mathrm{~min}$, then centrifuged at $10,000 \mathrm{rpm}$ for $10 \mathrm{~min}$ and to the supernatant, equal volume of chloroform: isoamyl alcohol (24:1) and $1 / 20$ th volume of $3 \mathrm{M}$ sodium acetate ( $\mathrm{pH} 4.8$ ) was added. Again centrifuging at $10,000 \mathrm{rpm}$ for $10 \mathrm{~min}$ and to the supernatant, 3 volumes of cold absolute alcohol were added. The precipitated DNA was separated by centrifugation and the pellet was dried and dissolved in TAE buffer (10 mM tris pH 8.0,1 mM EDTA) and stored in cold condition.

2.2.2.2. Agarose gel electrophoresis. Cleavage products were analyzed by agarose gel electrophoresis method. Test samples $(1 \mathrm{mg} / \mathrm{ml}$ ) were prepared in DMSO. The samples were added to the isolated DNA of E. coli. The samples were incubated for $2 \mathrm{~h}$ at $37^{\circ} \mathrm{C}$ and then $20 \mathrm{~mL}$ of DNA sample (mixed with bromo phenol blue dye at $1: 1$ ratio) was loaded carefully into the electrophoresis chamber wells along with standard DNA marker containing TAE buffer $(4.84 \mathrm{~g}$ tris base, pH 8.0, 0.5 M EDTA/1 L) and finally loaded on agarose gel and passed the constant $50 \mathrm{~V}$ of electricity for around $30 \mathrm{~min}$. Removing the gel and being stained with $30.0 \mathrm{mg} / \mathrm{ml}$ Acridine orange for $30 \mathrm{~min}$, The stain was removed from the pan by hot running tap water for 5-10 min. Agarose gels was sufficiently de-stained after $1 \mathrm{hr}$, the bands were observed under UV Transilluminator $\lambda$ Max $254 \mathrm{~nm}$. Photograph was taken by cannon digital camera the photograph used to determine the extent of DNA cleavage. Henceforth the results were compared with standard DNA marker. ${ }^{15}$

\subsubsection{Brine-shrimp lethality assay}

Brine-shrimp toxicity assay was used to determine cytotoxicity levels of the most active compounds $5 \mathrm{E}$ ( $\mathrm{a} \& \mathrm{~b}$ ). Each test compound was dissolved in DMSO to obtain the stock concentration of $1000 \mu \mathrm{g} / \mathrm{ml}$ and then stock solution was diluted to various concentrations $(1000-1.953 \mu \mathrm{g} / \mathrm{ml})$. In order to prevent the toxicity results from possible false effect originated from DMSO's toxicity, stock solutions of the compounds were prepared according to suggested volume range by dissolving $1 \mathrm{mg}$ of test compound in $10 \mu \mathrm{L}$ DMSO and completing to $1000 \mu \mathrm{l}$ with artificial seawater. Pure DMSO was used as a positive control for the toxicity assay. Fresh eggs of Artemia salina were hatched in a conical flask containing $300 \mathrm{ml}$ artificial seawater made by dissolving a commercial marine salt in deionized water. The flasks were well aerated with the aid of an air pump, and kept in a water bath at $25-30^{\circ} \mathrm{C}$. The larvae were hatched with in $48 \mathrm{~h}$. Ten larvae were transferred with pipette into each vial containing test compound and artificial seawater. A check count was performed after $24 \mathrm{~h}$ of exposure at room temperature and the number of dead larvae, exhibiting no internal or external movement during several seconds of observation, was noted. Three independent experiments were performed for each concentration of compounds. ${ }^{16}$

\section{Results and discussion}

\subsection{Chemistry}

The mannich bases of benzimidazole derivatives were synthesized by using the method described in earlier literature. ${ }^{13}$ All the compounds were obtained in good quantities. The condensation of the ortho-phenylene diamine (OPDA) and acids such as formic acid, acetic acid, benzoic acid, salicylic acid, sulpho salicylic acid, oxalic acid and pthalic acid yielded benzimidazole derivatives 3 (A-G). Targeted mannich bases compound [5(A-G)a,b] was obtained by a solution of 2-substituted benzimidazole $(0.005 \mathrm{~mol})$ in $10 \mathrm{ml}$ ethanol, $0.005 \mathrm{~mol}$ of secondary amine and $0.005 \mathrm{~mol}$ of formaldehyde and then the reaction mixture was refluxed for $8 \mathrm{~h}$. The completion of the reaction was confirmed by TLC. The melting point of the synthesized compounds was measured by using open capillary tube method. All the synthesized compounds gave satisfactory IR and ${ }^{1} \mathrm{H}$ NMR spectra were consistent with the assigned structures. Synthesis procedure of the benzimidazole derivatives was outlined in Schemes 1 and 2. The physicochemical data of the compounds are presented in Table 1.

\subsection{Physical and spectral data of synthesized compounds}

3.2.1. 1-(1H-Benzimidazol-1-yl)-N,N-dimethylmethanamine $5 A(a)$

$\mathrm{C}_{10} \mathrm{H}_{13} \mathrm{~N}_{3}, 66 \%$ yield, m.p. $170-173^{\circ} \mathrm{C}$, IR $\left(\mathrm{KBr}, \cup \mathrm{cm}^{-1}\right), 1201\{\mathrm{C}-\mathrm{N}$ str., (alkyl.)\}, 1272, $1364\{\mathrm{C}-\mathrm{N}$ str., (aryl.) $\}, 1477\left\{\mathrm{CH}_{2}\right.$ (bend.) $\}, 1770$ $\{\mathrm{C}=\mathrm{N}\}, 1587\{\mathrm{C}=\mathrm{C}\}, 751\{\mathrm{C}-\mathrm{H}$ (alkyl.) $\},{ }^{1} \mathrm{H}$ NMR (400 MHz, DMSO$\mathrm{d} 6, \delta \mathrm{ppm}), 2.27\left(\mathrm{~s}, 6 \mathrm{H},-\mathrm{CH}_{3}\right), 4.80\left(\mathrm{~s}, 2 \mathrm{H},-\mathrm{CH}_{2}-\right), 7.26-8.08(\mathrm{~m}$, $5 \mathrm{H}, \mathrm{Ar}-\mathrm{H})$.

\subsection{2. $\mathrm{N}$-(1H-Benzimidazol-1-ylmethyl)-N-ethylethanamine $5 A(b)$}

$\mathrm{C}_{12} \mathrm{H}_{17} \mathrm{~N}_{3}, 67.5 \%$ yield, m.p. $178-180{ }^{\circ} \mathrm{C}$, IR $\left(\mathrm{KBr}, \cup \mathrm{cm}^{-1}\right), 1201$ \{C-N str., (alkyl.)\}, $1477\left\{\mathrm{CH}_{2}\right.$ (bend.)\}, $1770\{\mathrm{C}=\mathrm{N}\}, 1587\{\mathrm{C}=\mathrm{C}\}$, $751\left\{\mathrm{C}-\mathrm{H}\right.$ (alkyl.)\}, ${ }^{1} \mathrm{H}$ NMR (400 MHz, DMSO-d6, $\left.\delta \mathrm{ppm}\right), 1.00(\mathrm{t}$, $\left.6 \mathrm{H},-\mathrm{CH}_{3}\right), 2.40\left(\mathrm{~m}, 4 \mathrm{H},-\mathrm{CH}_{2}-\right), 4.82\left(\mathrm{~s}, 2 \mathrm{H},-\mathrm{CH}_{2}-\right), 7.26-8.08(\mathrm{~m}$, $5 \mathrm{H}, \mathrm{Ar}-\mathrm{H})$.

\subsubsection{N,N-Dimethyl-1-(2-methyl-1H-benzimidazol-1-yl) methanamine $5 B(a)$}

$\mathrm{C}_{11} \mathrm{H}_{15} \mathrm{~N}_{3}, 68 \%$ yield, m.p. $262-264{ }^{\circ} \mathrm{C}, \mathrm{IR}\left(\mathrm{KBr}, \cup \mathrm{cm}^{-1}\right), 1012\{\mathrm{C}-\mathrm{N}$ str., (alkyl.)\}, 1286, 1157\{C-N str., (aryl.)\}, 1454\{ $\mathrm{CH}_{2}$ (bend.)\}, 1403 $\left\{\mathrm{CH}_{3}\right.$ (str.) $\}, 1644\{\mathrm{C}=\mathrm{N}\}, 1556,1573\{\mathrm{C}=\mathrm{C}\}, 745\{\mathrm{C}-\mathrm{H}$ (alkyl.) $\},{ }^{1} \mathrm{H}$ NMR (400 MHz, DMSO-d6, $\delta \mathrm{ppm}) 2.27-2.42\left(\mathrm{~s}, 9 \mathrm{H},-\mathrm{CH}_{3}\right)$, 4.78(s, $\left.2 \mathrm{H},-\mathrm{CH}_{2}-\right)$, 7.26-8.08(m, $\left.4 \mathrm{H}, \mathrm{Ar}-\mathrm{H}\right)$.

\subsection{4. l-N-[(2-Methyl-1H-benzimidazol-1-yl)methyl]ethanamine} $5 B(b)$

$\mathrm{C}_{13} \mathrm{H}_{19} \mathrm{~N}_{3}, 67 \%$ yield, m.p. $230-233^{\circ} \mathrm{C}$, IR $\left(\mathrm{KBr}, \cup \mathrm{cm}^{-1}\right), 1236\{\mathrm{C}-\mathrm{N}$ str., (alkyl.)\}, $1287\left\{\mathrm{C}-\mathrm{N}\right.$ str., (aryl.)\}, $1404\left\{\mathrm{CH}_{2}\right.$ (bend.)\}, $1352\left\{\mathrm{CH}_{3}\right.$ (str.) $\}, 1647\{\mathrm{C}=\mathrm{N}\}, 1522,1544\{\mathrm{C}=\mathrm{C}\}, 745\{\mathrm{C}-\mathrm{H}$ str., (alkyl) $\},{ }^{1} \mathrm{H}$ NMR (400 MHz, DMSO-d6, $\delta \mathrm{ppm}), 1.00\left(\mathrm{t}, 6 \mathrm{H}, \mathrm{CH}_{3}\right), 2.40\left(\mathrm{~m}, 4 \mathrm{H},-\mathrm{CH}_{2}\right)$, 2.42(s, $\left.3 \mathrm{H},-\mathrm{CH}_{3}\right), 4.80\left(\mathrm{~s}, 2 \mathrm{H},-\mathrm{CH}_{2}-\right), 7.26-8.08(\mathrm{~m}, 4 \mathrm{H}, \mathrm{Ar}-\mathrm{H})$.

\subsubsection{N,N-Dimethyl-1-(2-phenyl-1H-benzimidazol-1-yl) methanamine $5 \mathrm{C}(\mathrm{a})$}

$\mathrm{C}_{16} \mathrm{H}_{17} \mathrm{~N}_{3}, 65 \%$ yield, m.p. $326-328^{\circ} \mathrm{C}, \mathrm{IR}\left(\mathrm{KBr}, \cup \mathrm{cm}^{-1}\right), 1271\{\mathrm{C}-\mathrm{N}$ str., (aryl.)\}, 1436 $\left\{\mathrm{CH}_{2}\right.$ (bend.) $\}, 1601\{\mathrm{C}=\mathrm{N}\}, 711\{\mathrm{C}-\mathrm{H}$ (alkyl.) $\}$, ${ }^{1} \mathrm{H}$ NMR (400 MHz, DMSO-d6, $\left.\delta \mathrm{ppm}\right), 2.27\left(\mathrm{~s}, 6 \mathrm{H},-\mathrm{CH}_{3}\right), 4.80(\mathrm{~s}$, $\left.2 \mathrm{H},-\mathrm{CH}_{2}-\right), 7.26-7.70(\mathrm{~m}, 9 \mathrm{H}, \mathrm{Ar}-\mathrm{H})$.

\subsection{6. $N$-Ethyl-N-[(2-phenyl-1H-benzimidazol-1-yl)methyl] ethanamine $5 C(b)$}

$\mathrm{C}_{18} \mathrm{H}_{21} \mathrm{~N}_{3}, 65.5 \%$ yield, m.p. $315-318{ }^{\circ} \mathrm{C}$, IR $\left(\mathrm{KBr}, \cup \mathrm{cm}^{-1}\right), 1011$ \{C-N str., (alkyl.)\}, 1272\{C-N str., (aryl.)\}, 1434\{ $\mathrm{CH}_{2}$ (bend.)\}, 1600 $\{\mathrm{C}=\mathrm{N}\}, \quad 1555\{\mathrm{C}=\mathrm{C}\}, \quad 712.28\{\mathrm{C}-\mathrm{H}($ alkyl $)\},{ }^{1} \mathrm{H}$ NMR $(400 \mathrm{MHz}$, DMSO-d6, $\delta \mathrm{ppm}), 1.00\left(\mathrm{t}, 6 \mathrm{H},-\mathrm{CH}_{3}\right), 2.40\left(\mathrm{~m}, 4 \mathrm{H},-\mathrm{CH}_{2}-\right)$, $4.80\left(\mathrm{~s}, 2 \mathrm{H},-\mathrm{CH}_{2}-\right), 7.26-7.70(\mathrm{~m}, 9 \mathrm{H}, \mathrm{Ar}-\mathrm{H})$.

\subsubsection{2-\{1-[(Dimethylamino)methyl $]-1 H$-benzimidazol-2-yl $\}$ phenol $5 D(a)$}

$\mathrm{C}_{16} \mathrm{H}_{17} \mathrm{ON}_{3}, 63.7 \%$ yield, m.p. $256-259{ }^{\circ} \mathrm{C}$, IR $\left(\mathrm{KBr}, \cup \mathrm{cm}^{-1}\right), 1345$ $\{\mathrm{C}-\mathrm{N}$ str., (aryl.) $\}, 1413\left\{\mathrm{CH}_{2}\right.$ (bend.) $\}, 1627\{\mathrm{C}=\mathrm{N}\}, 1485\{\mathrm{C}=\mathrm{C}\}$, $751\left\{\mathrm{C}-\mathrm{H}\right.$ (alkyl.)\}, ${ }^{1} \mathrm{H}$ NMR (400 MHz, DMSO-d6, $\left.\delta \mathrm{ppm}\right), 2.27$ (s, $\left.6 \mathrm{H},-\mathrm{CH}_{3}\right), 4.80\left(\mathrm{~s}, 2 \mathrm{H},-\mathrm{CH}_{2}-\right), 5.00(\mathrm{~s}, 1 \mathrm{H},-\mathrm{OH}), 6.88-7.70(\mathrm{~m}, 8 \mathrm{H}$, Ar-H).

\subsubsection{2-\{1-[(Diethylamino $)$ methyl $]-1 H$-benzimidazol-2-yl $\}$ phenol $5 D(b)$}

$\mathrm{C}_{18} \mathrm{H}_{21} \mathrm{~N}_{3} \mathrm{O}, 64 \%$ yield, m.p. $260-262{ }^{\circ} \mathrm{C}$, IR $\left(\mathrm{KBr}, \cup \mathrm{cm}^{-1}\right), 1217$ $\left\{\mathrm{C}-\mathrm{N}\right.$ Str., (alkyl.)\}, 1270, $1345\{\mathrm{C}-\mathrm{N}$ str., (aryl.) $\}, 1460\left\{\mathrm{CH}_{2}\right.$ (bend.)\}, 
<smiles>[R]c1nc2ccccc2[nH]1</smiles>

$3(A-G)$<smiles>[R]NC</smiles>

$4(a, b)$<smiles>[R]c1nc2ccccc2n1CN([R])[R]</smiles>

$5(A-G) a, b$

$\mathrm{R}^{\prime}=\mathrm{a}=-\mathrm{CH}_{3}, \quad \mathrm{R}^{\prime}=\mathrm{b}=-\mathrm{C}_{2} \mathrm{H}_{5}$,

Synthesized compounds<smiles>[R]c1nc2ccccc2n1CN([R])[R]</smiles>

\begin{tabular}{llll}
\hline Compound s & $\mathbf{R}$ & $\mathbf{R}^{1}$ & $\mathbf{R}^{2}$ \\
\hline $5 \mathrm{~A}(\mathrm{a})$ & $-\mathrm{H}$ & $-\mathrm{CH}_{3}$ & $-\mathrm{CH}_{3}$ \\
$5 \mathrm{~A}(\mathrm{~b})$ & $-\mathrm{H}$ & $-\mathrm{C}_{2} \mathrm{H}_{5}$ & $-\mathrm{C}_{2} \mathrm{H}_{5}$ \\
$5 \mathrm{~B}(\mathrm{a})$ & $-\mathrm{CH}_{3}$ & $-\mathrm{CH}_{3}$ & $-\mathrm{CH}_{3}$ \\
$5 \mathrm{~B}(\mathrm{~b})$ & $-\mathrm{CH}_{3}$ & $-\mathrm{C}_{2} \mathrm{H}_{5}$ & $-\mathrm{C}_{2} \mathrm{H}_{5}$ \\
$5 \mathrm{C}(\mathrm{a})$ & $-\mathrm{C}_{6} \mathrm{H}_{5}$ & $-\mathrm{CH}_{3}$ & $-\mathrm{CH}_{3}$ \\
$5 \mathrm{C}(\mathrm{b})$ & $-\mathrm{C}_{6} \mathrm{H}_{5}$ & $-\mathrm{C}_{2} \mathrm{H}_{5}$ & $-\mathrm{C}_{2} \mathrm{H}_{5}$ \\
$5 \mathrm{D}(\mathrm{a})$ & $-\mathrm{C}_{6} \mathrm{H}_{4}(2-\mathrm{OH})$ & $-\mathrm{CH}_{3}$ & $-\mathrm{CH}_{3}$ \\
$5 \mathrm{D}(\mathrm{b})$ & $-\mathrm{C}_{6} \mathrm{H}_{4}(2-\mathrm{OH})$ & $-\mathrm{C}_{2} \mathrm{H}_{5}$ & $-\mathrm{C}_{2} \mathrm{H}_{5}$ \\
$5 \mathrm{E}(\mathrm{a})$ & $-\mathrm{C}_{6} \mathrm{H}_{3}(2-\mathrm{OH})\left(5-\mathrm{SO}_{2} \mathrm{OH}\right)$ & $-\mathrm{CH}_{3}$ & $-\mathrm{CH}_{3}$ \\
$5 \mathrm{E}(\mathrm{b})$ & $-\mathrm{C}_{6} \mathrm{H}_{3}(2-\mathrm{OH})\left(5-\mathrm{SO}_{2} \mathrm{OH}\right)$ & $-\mathrm{C}_{2} \mathrm{H}_{5}$ & $-\mathrm{C}_{2} \mathrm{H}_{5}$ \\
$5 \mathrm{~F}(\mathrm{a})$ & $-\mathrm{COOH}$ & $-\mathrm{CH}_{3}$ & $-\mathrm{CH}_{3}$ \\
$5 \mathrm{~F}(\mathrm{~b})$ & $-\mathrm{COOH}$ & $-\mathrm{C}_{2} \mathrm{H}_{5}$ & $-\mathrm{C}_{2} \mathrm{H}_{5}$ \\
$5 \mathrm{G}(\mathrm{a})$ & $-\mathrm{C}_{6} \mathrm{H}_{4}(2-\mathrm{COOH})$ & $-\mathrm{CH}_{3}$ & $-\mathrm{CH}_{3}$ \\
$5 \mathrm{G}(\mathrm{b})$ & $-\mathrm{C}_{6} \mathrm{H}_{4}(2-\mathrm{COOH})$ & $-\mathrm{C}_{2} \mathrm{H}_{5}$ & $-\mathrm{C}_{2} \mathrm{H}_{5}$ \\
\hline
\end{tabular}

Scheme 2. Preparation of 2- Substituted Benzimidazole derivatives from Ortho pheneylene diamine.

$1626\{\mathrm{C}=\mathrm{N}\}, 1484,1556\{\mathrm{C}=\mathrm{C}\}, 702\{\mathrm{C}-\mathrm{O}$ (str.), $752\{\mathrm{C}-\mathrm{H}$ (alkyl.) $\}$, ${ }^{1} \mathrm{H}$ NMR (400 MHz, DMSO-d6, $\left.\delta \mathrm{ppm}\right), 1.00\left(\mathrm{t}, 6 \mathrm{H},-\mathrm{CH}_{3}\right), 2.40(\mathrm{~m}$, $\left.4 \mathrm{H},-\mathrm{CH}_{2}-\right), 4.80\left(\mathrm{~s}, 2 \mathrm{H},-\mathrm{CH}_{2}-\right), 5.00(\mathrm{~s}, 1 \mathrm{H},-\mathrm{OH}), 6.88-7.70(\mathrm{~m}, 8 \mathrm{H}$, Ar-H).

Table 1

Physicochemical parameters of 2-substituted Benzimidazole derivatives.

\begin{tabular}{llllll}
\hline $\begin{array}{l}\text { Compound } \\
\text { name }\end{array}$ & $\begin{array}{l}\text { Molecular } \\
\text { formula }\end{array}$ & $\begin{array}{l}\text { Molecular } \\
\text { weight (gms) }\end{array}$ & $\begin{array}{l}\text { Percentage } \\
\text { yield }\end{array}$ & $\mathrm{R}_{\mathrm{f}}$ value & $\begin{array}{l}\text { Melting } \\
\text { point }\left({ }^{\circ} \mathrm{C}\right)\end{array}$ \\
\hline $5 \mathrm{~A}(\mathrm{a})$ & $\mathrm{C}_{10} \mathrm{H}_{13} \mathrm{~N}_{3}$ & 175.23 & $66 \%$ & 0.766 & $170-173$ \\
$5 \mathrm{~A}(\mathrm{~b})$ & $\mathrm{C}_{12} \mathrm{H}_{17} \mathrm{~N}_{3}$ & 203.283 & $67.5 \%$ & 0.865 & $178-180$ \\
$5 \mathrm{~B}(\mathrm{a})$ & $\mathrm{C}_{11} \mathrm{H}_{15} \mathrm{~N}_{3}$ & 189.26 & $68 \%$ & 0.903 & $262-264$ \\
$5 \mathrm{~B}(\mathrm{~b})$ & $\mathrm{C}_{13} \mathrm{H}_{19} \mathrm{~N}_{3}$ & 217.31 & $67 \%$ & 0.838 & $230-233$ \\
$5 \mathrm{C}(\mathrm{a})$ & $\mathrm{C}_{16} \mathrm{H}_{17} \mathrm{~N}_{3}$ & 251.33 & $65 \%$ & 0.856 & $326-328$ \\
$5 \mathrm{C}(\mathrm{b})$ & $\mathrm{C}_{18} \mathrm{H}_{21} \mathrm{~N}_{3}$ & 279.38 & $65.5 \%$ & 0.677 & $315-318$ \\
$5 \mathrm{D}(\mathrm{a})$ & $\mathrm{C}_{16} \mathrm{H}_{17} \mathrm{ON}_{3}$ & 267.33 & $63.7 \%$ & 0.806 & $256-259$ \\
$5 \mathrm{D}(\mathrm{b})$ & $\mathrm{C}_{18} \mathrm{H}_{21} \mathrm{~N}_{3} \mathrm{O}$ & 295.38 & $64 \%$ & 0.75 & $260-262$ \\
$5 \mathrm{E}(\mathrm{a})$ & $\mathrm{C}_{16} \mathrm{H}_{17} \mathrm{~N}_{3} \mathrm{O}_{4} \mathrm{~S}$ & 347.39 & $66.3 \%$ & 0.766 & $225-227$ \\
$5 \mathrm{E}(\mathrm{b})$ & $\mathrm{C}_{18} \mathrm{H}_{21} \mathrm{~N}_{3} \mathrm{O}_{4} \mathrm{~S}$ & 375.44 & $67 \%$ & 0.838 & $230-234$ \\
$5 \mathrm{~F}(\mathrm{a})$ & $\mathrm{C}_{11} \mathrm{H}_{13} \mathrm{~N}_{3} \mathrm{O}_{2}$ & 219.24 & $66 \%$ & 0.863 & $245-247$ \\
$5 \mathrm{~F}(\mathrm{~b})$ & $\mathrm{C}_{13} \mathrm{H}_{17} \mathrm{~N}_{3} \mathrm{O}_{2}$ & 247.29 & $65.5 \%$ & 0.872 & $258-261$ \\
$5 \mathrm{G}(\mathrm{a})$ & $\mathrm{C}_{17} \mathrm{H}_{17} \mathrm{~N}_{3} \mathrm{O}_{2}$ & 295.33 & $67.5 \%$ & 0.834 & $276-279$ \\
$5 \mathrm{G}(\mathrm{b})$ & $\mathrm{C}_{19} \mathrm{H}_{21} \mathrm{~N}_{3} \mathrm{O}_{2}$ & 323.38 & $66.5 \%$ & 0.829 & $298-301$ \\
\hline
\end{tabular}

3.2.9. 3-\{1-[(Dimethylamino) methyl]-1H-benzimidazol-2-yl\}-4hydroxy benzene sulfonicacid $5 E(a)$

$\mathrm{C}_{16} \mathrm{H}_{17} \mathrm{~N}_{3} \mathrm{O}_{4} \mathrm{~S}, 66.3 \%$, m.p. $225-227{ }^{\circ} \mathrm{C}$, IR $\left(\mathrm{KBr}, \cup \mathrm{cm}^{-1}\right), 1041$, $1157\left\{\mathrm{C}-\mathrm{N}\right.$ str., (alkyl.)\}, 1220, $1261\left\{\mathrm{C}-\mathrm{N}\right.$ str., (aryl.)\}, $1415\left\{\mathrm{CH}_{2}\right.$ (bend.)\}, $1647\{\mathrm{C}=\mathrm{N}\}, 1480\{\mathrm{C}=\mathrm{C}\}, 1346\{\mathrm{~S}=\mathrm{O}\}, 1296\{\mathrm{C}-\mathrm{O}$ (str.)\}, $734\left\{\mathrm{C}-\mathrm{H}\right.$ (alkyl.) \}, ${ }^{1} \mathrm{H}$ NMR (400 MHz, DMSO-d6, $\delta \mathrm{ppm}$ ), 2.00(s, $1 \mathrm{H}$, $\left.-\mathrm{SO}_{3} \mathrm{H}\right), 2.27\left(\mathrm{~s}, 6 \mathrm{H},-\mathrm{CH}_{3}\right), 4.80\left(\mathrm{~s}, 2 \mathrm{H},-\mathrm{CH}_{2}-\right), 5.00\left(\mathrm{~s}, 1 \mathrm{H},-\mathrm{SO}_{3} \mathrm{H}\right)$, 7.07-7.98(m, 7H, Ar-H).

3.2.10. 3-\{1-[(Diethylamino) methyl] $-1 H$ - benzimidazol-2-yl\}-4hydroxy benzene sulfonicacid $5 E(b)$

$\mathrm{C}_{18} \mathrm{H}_{21} \mathrm{~N}_{3} \mathrm{O}_{4} \mathrm{~S}, 67 \%$, m.p. $230-234^{\circ} \mathrm{C}$, IR $\left(\mathrm{KBr}, \cup \mathrm{cm}^{-1}\right), 1040,1157$ $\left\{\mathrm{C}-\mathrm{N}\right.$ str., (alkyl.)\}, 1220, $1261\left\{\mathrm{C}-\mathrm{N}\right.$ str., (aryl.)\}, $1437\left\{\mathrm{CH}_{2}\right.$ (bend.)\}, $1647\{\mathrm{C}=\mathrm{N}\}, 1480\{\mathrm{C}=\mathrm{C}\}, 1296\{\mathrm{~S}=\mathrm{O}\}, 674\{\mathrm{C}-\mathrm{H}$ (alkyl.) $\},{ }^{1} \mathrm{H}$ NMR (400 MHz, DMSO-d6, $\delta \mathrm{ppm}), 1.00\left(\mathrm{t}, 6 \mathrm{H},-\mathrm{CH}_{3}\right), 2.00\left(\mathrm{~s}, 1 \mathrm{H},-\mathrm{SO}_{3} \mathrm{H}\right)$, $2.40\left(\mathrm{~m}, 4 \mathrm{H},-\mathrm{CH}_{2}-\right), 4.80\left(\mathrm{~s}, 2 \mathrm{H},-\mathrm{CH}_{2}-\right), 5.00\left(\mathrm{~s}, 1 \mathrm{H},-\mathrm{SO}_{3} \mathrm{H}\right)$, 7.07-7.98(m, 7H, Ar-H).

\subsubsection{1-[(Dimethylamino)methyl]-1H-Benzimidazole-2-carboxylic} acid $5 F(a)$

$\mathrm{C}_{11} \mathrm{H}_{13} \mathrm{~N}_{3} \mathrm{O}_{2}, 66 \%$ yield, m.p. $245-247^{\circ} \mathrm{C}$, IR $\left(\mathrm{KBr}, \mathrm{u} \mathrm{cm}^{-1}\right), 1120$ \{C-N str., (alkyl.)\}, 1383\{C-N str., (aryl.)\}, $724\left\{\mathrm{CH}_{2}\right.$ (bend.) $\}, 3036$ 
$\{\mathrm{O}-\mathrm{H}\}, 1766\{\mathrm{C}-\mathrm{O}$ (str.) $\}, 1544\{\mathrm{~N}-\mathrm{H}\}, 2874\{\mathrm{C}-\mathrm{H}$ (str.) $\},{ }^{1} \mathrm{H}$ NMR (400 MHz, DMSO-d6, $\delta \mathrm{ppm}), 2.27\left(\mathrm{~s}, 6 \mathrm{H},-\mathrm{CH}_{3}\right), 4.80(\mathrm{~s}, 2 \mathrm{H},-$ $\left.\mathrm{CH}_{2}-\right), 7.26-7.70(\mathrm{~m}, 4 \mathrm{H}, \mathrm{Ar}-\mathrm{H}), 11(\mathrm{~s}, 1 \mathrm{H},-\mathrm{COOH})$.

\subsubsection{1-[(Diethylamino)methyl]-1H-Benzimidazole-2-carboxylic acid $5 F(b)$}

$\mathrm{C}_{13} \mathrm{H}_{17} \mathrm{~N}_{3} \mathrm{O}_{2}, 65.5 \%$ yield, m.p. $258-261{ }^{\circ} \mathrm{C}$, IR $\left(\mathrm{KBr}, \cup \mathrm{cm}^{-1}\right), 1121$ \{C-N str., (alkyl.)\}, $1383\{\mathrm{C}-\mathrm{N}$ str., (aryl.) $\}, 728\left\{\mathrm{CH}_{2}\right.$ (bend.) $\}, 1548$ $\{\mathrm{N}-\mathrm{H}$ (bend.) $\}, 3043\{\mathrm{O}-\mathrm{H}\}, 1666\{\mathrm{C}=\mathrm{O}\}, 2875\{\mathrm{C}-\mathrm{H}$ (alkyl. $)\},{ }^{1} \mathrm{H}$ NMR (400 MHz, DMSO-d6, $\delta \mathrm{ppm}), 1.00\left(\mathrm{t}, 6 \mathrm{H},-\mathrm{CH}_{3}\right), 2.40\left(\mathrm{~m}, 4 \mathrm{H},-\mathrm{CH}_{2}-\right.$ ), $4.80\left(\mathrm{~s}, 2 \mathrm{H},-\mathrm{CH}_{2}-\right), 7.26-7.70(\mathrm{~m}, 4 \mathrm{H}, \mathrm{Ar}-\mathrm{H}), 11(\mathrm{~s}, 1 \mathrm{H},-\mathrm{COOH})$.

\subsubsection{2-\{1-[(Dimethylamino) methyl $]-1 H$-benzimidazol-2-yl $\}$}

benzoic acid $5 \mathrm{G}(\mathrm{a})$

$\mathrm{C}_{17} \mathrm{H}_{17} \mathrm{~N}_{3} \mathrm{O}_{2}, 67.5 \%$ yield, m.p. $276-279{ }^{\circ} \mathrm{C}$, IR $\left(\mathrm{KBr}, \cup \mathrm{cm}^{-1}\right), 1131$ $\{\mathrm{C}-\mathrm{N}$ str., (alkyl.) $\}, 1401\{\mathrm{C}-\mathrm{N}$ str., (aryl.) $\}, 742\left\{\mathrm{CH}_{2}\right.$ (bend.) $\}, 3340$ $\{\mathrm{O}-\mathrm{H}\}, 1736\{\mathrm{C}-\mathrm{O}$ (str.) $\}, 1645\{\mathrm{~N}-\mathrm{H}\},{ }^{1} \mathrm{H}$ NMR (400 MHz, DMSO-d6, $\delta \mathrm{ppm}), 2.27\left(\mathrm{~s}, 6 \mathrm{H},-\mathrm{CH}_{3}\right), 4.80\left(\mathrm{~s}, 2 \mathrm{H},-\mathrm{CH}_{2}-\right), 7.26-8.19(\mathrm{~m}, 8 \mathrm{H}, \mathrm{Ar}-$ $\mathrm{H}), 11(\mathrm{~s}, 1 \mathrm{H},-\mathrm{COOH})$

\subsubsection{2-\{1-[(Diethylamino)methyl $]-1 H$-benzimidazol-2-yl $\}$}

benzoic acid $5 G(b)$

$\mathrm{C}_{19} \mathrm{H}_{21} \mathrm{~N}_{3} \mathrm{O}_{2}, 66.5 \%$ yield, m.p. $298-301^{\circ} \mathrm{C}, \mathrm{IR}\left(\mathrm{KBr}, \cup \mathrm{cm}^{-1}\right), 1081$ $\left\{\mathrm{C}-\mathrm{N}\right.$ str., (alkyl.)\}, $1401\left\{\mathrm{C}-\mathrm{N}\right.$ (str.)\}, $741\left\{\mathrm{CH}_{2}\right.$ (bend.) $\}, 3333\{\mathrm{O}-\mathrm{H}\}$, $1548\left\{\mathrm{C}=\mathrm{C}\right.$ (str.)\}, ${ }^{1} \mathrm{H}$ NMR (400 MHz, DMSO-d6, $\left.\delta \mathrm{ppm}\right), 1.00(\mathrm{t}$, $\left.6 \mathrm{H},-\mathrm{CH}_{3}\right), 2.40\left(\mathrm{~m}, 4 \mathrm{H},-\mathrm{CH}_{2}-\right), 4.80\left(\mathrm{~s}, 2 \mathrm{H},-\mathrm{CH}_{2}-\right), 7.26-8.19(\mathrm{~m}$, $8 \mathrm{H}, \mathrm{Ar}-\mathrm{H}), 11(\mathrm{~s}, 1 \mathrm{H},-\mathrm{COOH})$.

\subsection{Biological activity}

The title compounds $[5(A-G)$ a,b] were evaluated for their in vitro antimicrobial activity against the following six human pathogenic microorganism Bacillus subtilis (NCIM 2458), Staphylococcus aureus (ATCC 25923), Escherichia coli (ATCC 15830), Salmonella typhi (ATCC 8713) Candida albicans (ATCC 76615), and Aspergillus niger (NCIM 1207) were tested for the antimicrobial efficiency of synthesized compounds. Ampicillin and fluconazole used as a standard drug for antibacterial and fungal study respectively. The observed antimicrobial data (MIC, MBC and MFC) of the compounds and the reference drugs are given in Tables 2 and 3.

When compared with the reference drug Ampicillin, most of the compounds in the series exhibited considerable antibacterial activity against gram-negative bacteria such as E. coli and S. Typhi

Table 2

In vitro Anti microbial Manich Base of 2-substituted Benzimidazole derivatives.

\begin{tabular}{|c|c|c|c|c|c|c|}
\hline Compound & A & B & C & D & $\mathbf{E}$ & $\mathbf{F}$ \\
\hline $5 \mathrm{~A}(\mathrm{a})$ & 128 & 128 & $>64$ & $>128$ & 512 & 512 \\
\hline $5 A(b)$ & 256 & 128 & 128 & 128 & $>512$ & 512 \\
\hline $5 \mathrm{~B}(\mathrm{a})$ & $>256$ & $>512$ & 128 & 256 & 128 & $>256$ \\
\hline $5 B(b)$ & 512 & $>256$ & 128 & $>128$ & 128 & $>256$ \\
\hline $5 C(a)$ & $>128$ & 256 & 256 & 512 & 512 & 512 \\
\hline $5 C(b)$ & 128 & 256 & $>256$ & $>256$ & 256 & $>256$ \\
\hline $5 \mathrm{D}(\mathrm{a})$ & 256 & 512 & 256 & 256 & 512 & 256 \\
\hline $5 \mathrm{D}(\mathrm{b})$ & 512 & $>256$ & 512 & $>128$ & $>256$ & 256 \\
\hline $5 \mathrm{E}(\mathrm{a})$ & 16 & 16 & 8 & 16 & 32 & 64 \\
\hline $5 \mathrm{E}(\mathrm{b})$ & 16 & $>8$ & 4 & $>8$ & $>16$ & $>32$ \\
\hline $5 \mathrm{~F}(\mathrm{a})$ & $>64$ & $>32$ & 64 & 32 & 128 & 64 \\
\hline $5 \mathrm{~F}(\mathrm{~b})$ & 64 & $>64$ & 64 & 32 & $>128$ & $>64$ \\
\hline $5 G(a)$ & $>256$ & 256 & $>128$ & $>256$ & $>512$ & $>256$ \\
\hline $5 G(b)$ & 512 & 256 & 256 & 256 & 512 & 512 \\
\hline Ampicillin & 2 & 2 & 1 & 1 & - & - \\
\hline Fluconazole & - & - & - & - & 1 & 2 \\
\hline
\end{tabular}

Note: A: Bacillus subtilis (NCIM 2458), B: Staphylococcus aureus (ATCC 25923), C: Escherichia coli (ATCC 15830), D: Salmonella typhi (ATCC 8713), E: Candida albicans (ATCC 76615), F: Aspergillus niger (NCIM 1207), E: Candida albicans (ATCC 76615), F: Aspergillus niger (NCIM 1207).
Table 3

The Minimum bacterial concentration (MBC) and Minimum fungal concentration (MFC) Manich Base of 2-substituted Benzimidazole derivatives.

\begin{tabular}{|c|c|c|c|c|c|c|}
\hline Compound & A & B & C & D & $\mathbf{E}$ & $\mathbf{F}$ \\
\hline $5 \mathrm{~A}(\mathrm{a})$ & 256 & 256 & 128 & 256 & 1024 & 1024 \\
\hline $5 \mathrm{~A}(\mathrm{~b})$ & 512 & 256 & 256 & 256 & 1024 & 1024 \\
\hline $5 B(a)$ & 512 & 1024 & 256 & 512 & 256 & 512 \\
\hline $5 B(b)$ & 1024 & 512 & 256 & 256 & 256 & 512 \\
\hline $5 C(a)$ & 256 & 512 & 512 & 1024 & 1024 & 1024 \\
\hline $5 C(b)$ & 256 & 512 & 512 & 512 & 512 & 512 \\
\hline $5 \mathrm{D}(\mathrm{a})$ & 512 & 1024 & 512 & 512 & 1024 & 512 \\
\hline $5 \mathrm{D}(\mathrm{b})$ & 1024 & 512 & 1024 & 256 & 512 & 512 \\
\hline $5 \mathrm{E}(\mathrm{a})$ & 32 & 128 & 16 & 32 & 64 & 128 \\
\hline $5 E(b)$ & 32 & 16 & 8 & 16 & 32 & 64 \\
\hline $5 \mathrm{~F}(\mathrm{a})$ & 128 & 64 & 128 & 64 & 256 & 128 \\
\hline $5 F(b)$ & 128 & 128 & 128 & 64 & 256 & 128 \\
\hline $5 G(a)$ & 512 & 512 & 256 & 512 & 1024 & 512 \\
\hline $5 G(b)$ & 1024 & 512 & 512 & 512 & 1024 & 1024 \\
\hline Ampicillin & 4 & 4 & 2 & 2 & - & - \\
\hline Fluconazole & - & - & - & - & 2 & 4 \\
\hline
\end{tabular}

Note: A: Bacillus subtilis (NCIM 2458), B: Staphylococcus aureus (ATCC 25923), C Escherichia coli (ATCC 15830), D: Salmonella typhi (ATCC 8713), E: Candida albicans (ATCC 76615), F: Aspergillus niger (NCIM 1207).

(Table 2). The compounds 5E (a) and (b) were more potent than reference against gram-negative bacteria. The MIC values of compound $5 \mathrm{E}$ (a) against E. coli $8 \mu \mathrm{g} / \mathrm{ml}$, S. Typhi $16 \mu \mathrm{g} / \mathrm{ml}, 5 \mathrm{E}$ (b) against E. coli $4 \mu \mathrm{g} / \mathrm{ml}$, and S. Typhi $8 \mu \mathrm{g} / \mathrm{ml}$. Among the compounds $5 \mathrm{E}$ (a) and (b) exhibited significant MBC values 16, 32 and 8, $16 \mu \mathrm{g} / \mathrm{ml}$ respectively against the gram-negative bacterial species. Antifungal activity of the tested mannich base benzimidazole derivatives was observed as significant growth inhibition against both funguses. Among the compounds 5E (a) and (b) were found to be higher MIC $(32,64$ and $16,32 \mu \mathrm{g} / \mathrm{ml})$ against $C$. albicans and A. niger respectively. The MFC were find highest in the compound $5 \mathrm{E}$ (b) $(33,64 \mu \mathrm{g} / \mathrm{ml})$ against the two tested fungal species respectively (Table 3 ).

From the results of antibacterial and antifungal screening, it was observed that of sulfo salicyl group of benzimidazole derivative $5 \mathrm{E}$ (a) and (b) exhibited dominating activity over the series. This may be attributed to their enhanced electronic character due to the presence of sulfonyl group at the benzimidazole residue favoring greater penetration through microbial membrane.

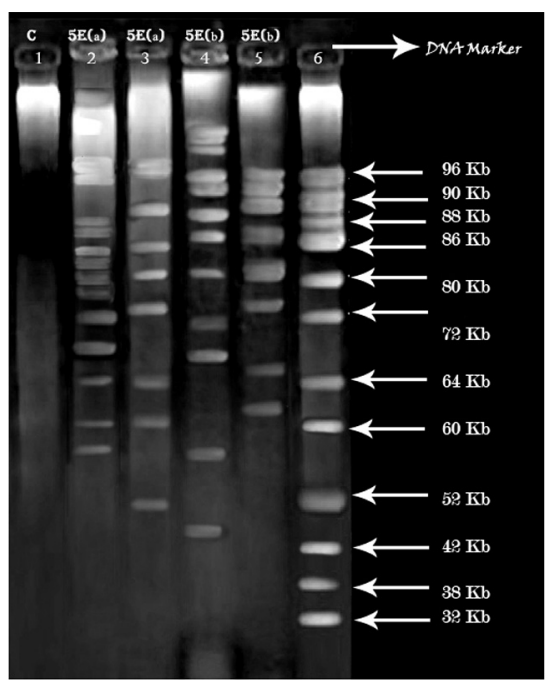

Note: The lane one serves as a control (DMSO) treated with DNA, there no cleavage induced by control. Lane 2 and 3 showed the DNA cleavage of compound $5 \mathrm{E}$ (a), the slicing occurring on the range from 96-52 kbs. The compound $5 \mathrm{E}$ (b) strongly cleavage the DNA without traces from $>96-43 \mathrm{kbs}$ in the lane 4 and 5 . The marker DNA placed on the lane no 6.

Fig. 1. DNA cleavage study for the compounds $5 E$ (a \& b). 
Table 4

Brine-shrimp toxicity results of the compounds $5 \mathrm{E}$ (a \& b).

\begin{tabular}{|c|c|c|}
\hline \multirow[t]{2}{*}{ Concentration $\mu \mathrm{g} / \mathrm{ml}$} & \multicolumn{2}{|l|}{ Mortality } \\
\hline & $5 \mathrm{E}(\mathrm{a})$ & $5 \mathrm{E}(\mathrm{b})$ \\
\hline 1000 & 4 & 4 \\
\hline 500 & 3 & 4 \\
\hline 250 & 3 & 3 \\
\hline 125 & 2 & 2 \\
\hline 62.5 & 1 & 1 \\
\hline 31.25 & 1 & 1 \\
\hline 15.625 & 1 & 1 \\
\hline 07.812 & 1 & 1 \\
\hline 03.906 & 1 & 1 \\
\hline 01.953 & 1 & 1 \\
\hline Control & 1 & 1 \\
\hline $\mathrm{LC}_{50}$ & $>1000$ & $>1000$ \\
\hline Toxicity level & No toxicity & No toxicity \\
\hline
\end{tabular}

The results of DNA cleavage (Fig. 1) for mannich base benzimidazole compounds studied by agarose gel electrophoresis method. The gel after electrophoresis clearly revealed that, compounds 5E (a) and (b) did cleave the DNA completely, as no traces of DNA were found. The most impressive cleavage feature observed for $5 \mathrm{E}(\mathrm{b})$. This is indicates that the compound $5 \mathrm{E}(\mathrm{b})$ is capable of performing direct double-strand scission, as a consequence, this compound serve better antimicrobial applications.

A chemical agent is valuable in medicinal field if only it possesses low toxicity with significant activity. Thus, toxicity of the compounds 5E (a) and (b) which have the highest antibacterial efficacy needs to be revealed. For this purpose Brine-Shrimp (Artemia salina) lethality assay was performed. This assay is regarded as a helpful method for preliminary screening of toxicity, and it has been used for establishing of microbial toxins, plant extract toxicity, heavy metals, and cytotoxicity testing of, natural and synthetic organic compounds. Moreover, A. salina toxicity test results show a good correlation with animals and human acute oral toxicity data. Likewise, the prognostic screening potential of the aquatic invertebrate tests for acute oral toxicity in man, including $A$. salina toxicity test, was slightly better than the rodent tests for test compounds. Toxicity test results calculate $\mathrm{LC}_{50}$ values and $95 \%$ confidence intervals. The mannich base benzimidazole compounds $5 \mathrm{E}$ (a) and (b) give $\mathrm{LC}_{50}$ values of the compounds and 95\% confidence intervals because number of dead larvae did not exceed $50 \%$ of total larvae. This was a significant result demonstrating that the tested compounds are non-toxic in the tested concentration range. Toxicity test results were presented in (Table 4).

\section{Conclusion}

A series of mannich bases of 2-substituted benzimidazole derivatives were synthesized and their structures were elucidated by spectral data. The preliminary in vitro antibacterial and, antifungal toxicological screening results of novel benzimidazole derivatives $[5(A-G)$ a,b] reported good to moderate antimicrobial activity. The compound $5 \mathrm{E}$ (a) and (b) exhibited broad spectrum of antibacterial activity and antifungal activity. Most effective compounds were found to be non-toxic A. salina toxicity test and cleave the E. coli genomic DNA completely, as no traces of DNA were found. With the suitable molecular modification, these compounds can prove as potent antimicrobial agents in future.

\section{Conflicts of interest}

All authors have none to declare.

\section{References}

1. Kalyankar TM, Pekamwar SS, Wadher SJ, Tiprale PS, Shinde GH. Review on benzimidazole derivative. Int J Che Pharm Sci. 2012;3:1-10.

2. Elerafi Mohamed G, Ibrahim Mohamed N. Synthesis and spectral studies of mannich bases derived from 2-substituted benzimidazoles. Int I Chem Tech Res. 2010;2:2097-2099.

3. Langtry HD, Wilde MI. Omeprazole: a review of its use in Helicobacter pylori infection, gastro-oesophageal reflux disease and peptic ulcers induced by nonsteroidal anti-inflammatory drugs. Drugs. 1998;56:447-486.

4. Hazelton JC, Iddon B, Suschitzky H, Woolley LH. Synthesis of polysubstituted ophenylenediamines and their conversion into heterocycles, particularly 2substituted benzimidazoles with known or potential anthelminthic activity. Tetrahedron. 1995;51:10771-10794.

5. Ansari KF, Lal C. Synthesis, physicochemical properties and antimicrobial activity of some new benzimidazole derivatives. Eur J Med Chem. 2009;44:40284033.

6. Kennis LEL, Vandenberk J, Boey JM. The chemical development of selective and specific serotonin S2-antagonists. Drug Dev Res. 1986;8:133-140.

7. Braun Stephan, Botzki Alexander, Salmen Sunnhild, et al. Design of benzimidazole- and benzoxazole-2-thione derivatives as inhibitors of bacterial hyaluronan lyase. Eur J Med Chem. 2011;46:4419-4429.

8. Achar KC, Hosamani KM, Seetharamareddy HR. In-vivo analgesic and anti-inflammatory activities of newly synthesized benzimidazole derivatives. Eur J Med Chem. 2010;45:2048-2054.

9. Zhang SL, Damu GL, Zhang L, Geng RX, Zhou CH. Synthesis and biological evaluation of novel benzimidazole derivatives and their binding behavior with bovine serum albumin. Eur J Med Chem. 2012;55:164-175.

10. Pandeya SN, Lakshmi VS, Patel A. Biological activity of mannich bases. Ind J Pharm Sci. 2003:65:213-221.

11. Vijayaraghavan S, Somani RR, Shirodkar PY, Kadam VJ. Microwave assisted synthesis and antimicrobial activity of some newer mannich bases. Int J Pharm Tech Res. 2009;4:811-815.

12. Pathak Devender, Siddiqui Nadeem, Bhrigu Bhanupriya, Ahsan Waquar, Shamshar Alam M. Benzimidazoles: a new profile of biological activities. Der Pharmacia Lett. 2010;2:27-34.

13. Al Messmary Mohamed, Gebriel Elarfil Mohamed, Mohamed Rahim. Synthesis and spectral studies of mannich bases derived from 2-substituted benzimidazoles. Int J Chem Tech Res. 2010;2:1714-1716.

14. Vijey Aanandhi M, Abhay Kumar Verma, Sujatha R, Kamal Raj R. Synthesis and characterization of novel mannich bases of benzimidazole derivatives for antibacterial and antifungal activity. Int J Pharm Pharm Sci. 2013;5:295-297.

15. Shingalapur Ramya V, Hosamani Kallappa M, Keri Rangappa S, Hugar Mallinath H. Derivatives of benzimidazole pharmacophore: synthesis, anticonvulsant, antidiabetic and DNA cleavage studies. Eur J Med Chem. 2010;45:1753-1759.

16. Ozkay Yusuf, Tunal Yagmur, Karac Hulya, Iskdag Ilhan. Antimicrobial activity and a SAR study of some novel benzimidazole derivatives bearing hydrazone moiety. Eur J Med Chem. 2010;45:3293-3298. 